\title{
Is sleep related to cancer in murine models of obstructive sleep disorder?
}

\section{To the Editors:}

The recent article by ALMENDros et al. [1] brings to light an interesting, but yet rarely discussed, relationship: the association between sleep disordered breathing and cancer. Overall, the study found that high-rate intermittent hypoxia (an animal model that mimics obstructive sleep apnoea) contributed to melanoma tumour growth in mice. There was an increase in the size and degree of necrosis, which were, on average, two times higher in mice exposed to intermittent hypoxia compared with the normoxia group. We would like to congratulate the authors for this study and emphasise the relevance of this research, as well as their results, by making some brief remarks. The issue addressed in this study, sleep apnoea and melanoma, has great clinical importance and relevance in a remarkable public health matter due to the high incidence and severity of both diseases. In general, sleep is a biological phenomenon characterised by a reversible and active state, and is part of a major physiological and psychological need of human beings. However, the prevalence of sleep disorders are increasing markedly, especially sleep apnoea [2]. The impact of sleep disorders on the health of humans has not been fully clarified; it is still the subject of research. Since sleep has a bidirectional relationship with most systems, changes in its pattern can generate negative consequences for the organism, as well as diseases can impair sleep. Studies have shown that sleep apnoea can impair the health of individuals affected by this disease [2], such as by causing cardiovascular complications [3]. Melanoma is a specific cancer that affects the epithelial tissue, and it can metastasise if not detected and treated in the early stages. Its incidence rate is high. For instance, in 1973, the incidence rate was 7.5 cases per 100,000 inhabitants and in 2002, this figure rose to 21.9 cases per 100,000 [4]. More recent data indicate that in 2012, we will see $\sim 76,250$ new cases of melanoma of the skin, causing 9,180 deaths on average [5]. Notably, sleep apnoea has also been a disease of great clinical relevance because it is a syndrome with a relative incidence and prevalence of $\sim 33 \%$ of the population [2]. Given the magnitude of the severity of apnoea and melanoma, we would like to highlight the clinical implications of the findings of AlmENDros et al. [1], as well as their contribution of this relevant scientific evidence regarding sleep and cancer. These findings provide further knowledge about the mechanisms involved in tumour progression associated with sleep apnoea. Furthermore, it shall be mentioned that, despite being conducted in laboratory animals, the background and discussion provided by the authors ensure a translational applicability to this study. In conclusion, this study highlights the importance of diagnosis and treatment of sleep apnoea, especially in patients with cancer; thus, a better prognosis and a better quality of life for patients can be achieved.

\section{K.T. Nozoe, S. Tufik and M.L. Andersen}

Departamento de Psicobiologia, Universidade Federal de São Paulo, São Paulo, Brazil.

Correspondence: M.L. Andersen, Departamento de Psicobiologia, Universidade Federal de São Paulo, Rua Napoleão de Barros 925, Vila Clementino, SP-04024-002, São Paulo, Brazil. E-mail: mandersen@unifesp.br

Statement of Interest: None declared.

\section{REFERENCES}

1 Almendros I, Monteserrat JM, Ramírez J, et al. Intermittent hypoxia enhances cancer progression in a mouse model of sleep apnea. Eur Respir J 2012; 39: 215-217.

2 Tufik S, Santos-Silva R, Taddei JA, et al. Obstructive sleep apnea syndrome in the Sao Paulo Epidemiologic Sleep Study. Sleep Med 2010; 11: 441-446.

3 Cintra F, Tufik S, D'Almeida V, et al. Cysteine: a potential biomarker of obstructive sleep apnea. Chest 2010; 139: 246-252.

4 Markovic SN, Erickson LA, Rao RD, et al. Malignant melanoma in the 21st century, part 1: epidemiology, risk factors, screening, prevention, and diagnosis. Mayo Clin Proc 2007; 82: 346-380.

5 Siegel R, Ward E, Brawley O, et al. The impact of eliminating socioeconomic and racial disparities on premature cancer deaths. CA Cancer J Clin 2011; 61: 212-236.

DOI: $10.1183 / 09031936.00003412$

\section{Primary lung adenocarcinoma: characteristics by smoking habit and sex}

\section{To the Editors:}

The recent article by NAgY-MignotTe et al. [1] focused on a relevant and still open issue about lung carcinogenesis, which is how sex and smoking habit could affect lung cancer onset and progression. Through an observational, retrospective, single-centre study, the authors analysed the epidemiological features of an extraordinarily large and well recorded cohort of 
primary lung adenocarcinomas. Their results are coherent with previously published reports that suggest a potential susceptibility of females to developing adenocarcinomas; interestingly, the study unveils different generations in tumour presentation of female patients according to their smoking status.

We read this article with great interest and we believe that it could be of clear interest in terms of preventive medicine. However, we would like to draw attention to the fact that cancer is a complex disease, associated with genetic lesions (mainly represented by changes in DNA sequence resulting in the production of altered proteins) that tend to accumulate over time as a result of exposure to environmental carcinogens, diet and lifestyle.

Lung cancer represents the leading cause of death for solid tumours worldwide with an annual mortality of $>1$ million. It really identifies an extremely heterogeneous group of disorders that feature different morphological and molecular assets. Indeed, new histopathological classifications are coherently addressed to a multidisciplinary classification of lung tumours, mainly of adenocarcinomas [2]. Although many of the genes that are involved in lung adenocarcinoma onset remain to be discovered, a number of tumour-activating somatic genetic lesions ("driving" lesions) have already been detected in a substantial fraction of patients. Post-genomic approaches and high-throughput technologies are addressing the key issue of how genome data could be translated into a system for detection and determination of the disease prognosis and prediction of drug responses. This point should be underlined when approaching a still-evolving landscape, such as lung adenocarcinoma [3].

From this perspective, the limitation of traditional epidemiology essentially emerges from the analysis of weak effects, and complex diseases and exposure circumstances. However, technical developments, as well as increasing knowledge on pathogenetic mechanisms, are advantages in the genetic/ molecular approach to epidemiology. Molecular epidemiology aims to identify human cancer risk as well as the incidence or outcome of disease based upon individual exposures and genetic susceptibilities to cancer [4]. Such studies are designed based on the knowledge that cancer is a multistage process where each stage is composed of several genetic events, and each event occurs because of multiple steps requiring carcinogen metabolic activation, detoxification, mutations in critical parts of cancer-related genes, and failure to repair DNA damage or undergo programmed cell death. Besides this, interindividual variation in carcinogen metabolism and associated responses should be investigated. Within respect to lung cancer, it is well known that the primary aetiology is tobacco smoking, but an understanding of why some smokers develop lung cancer, and others do not, remains unclear. Interestingly, although the work generally referred to lung cancer without specifying the histological type, a region on chromosome 15 has recently been identified that, if mutated, dramatically increases a smoker's risk of developing lung cancer, depending on the number of copies of the 15q24 susceptibility locus [5]. Also, existing studies suggest that females are more susceptible to the carcinogens in cigarettes and develop lung cancer after fewer years of smoking. Importantly, the concept of risk stratification among the female patient population clearly emerges from the present study. Indeed, its results showed on one hand, a greater susceptibility and cancer progression in young, currently smoking females and, on the other, demonstrated that never-smoker females are generally diagnosed with extended latency, which clinically implies a poor prognosis, independently of the use of targeted drugs. Overall, these data clearly highlight the need to improve both primary and secondary prevention screening.

NAGY-MignOTTE et al. [1] properly and accurately described the epidemiological phenomena; nevertheless, this kind of study does not allow the identification of a biomarker intended as a variable to be measured, either of an assay (the test aiming to measure the marker) or of a measurement (an individual result of the value of the marker) that could be exploited for a deeper investigation of the complex network between sex, smoking habit and susceptibility to lung cancer development. Sample variability, in terms of morphology, size and collection, is certainly a key issue that could impact data analysis, mainly in large epidemiological studies. We thus would like to underline that the efforts that are now ongoing to understand the role of genetic predisposition to lung cancer risk might not be directed toward adequate sample storage, banking and processing.

\section{G.M. Stella ${ }^{*, \#}$, M. Luisetti ${ }^{\#}$ and E. Pozzi}

*Dipartimento di Scienze Oncologiche - Istituto per la Ricerca e la Cura del Cancro (IRCC), University of Turin, Turin, ${ }^{\#}$ Clinica Malattie Apparato Respiratorio, University of Pavia, and IRCCS Policlinico San Matteo, Pavia, and "Policlinico di Monza, University of Pavia, Monza, Italy.

Correspondence: G.M. Stella, IRCCS Fondazione, Policlinico San Matteo, Pneumology, Piazzale Golgi 19, Pavia 27100, Italy. E-mail: g.stella@smatteo.pv.it

\section{Statement of Interest: None declared.}

\section{REFERENCES}

1 Nagy-Mignotte $\mathrm{H}$, Guillem $\mathrm{P}$, Vesin A, et al. Primary lung adenocarcinoma: characteristics by smoking habit and sex. Eur Respir J 2011; 38: 1412-1419.

2 Travis WD, Brambilla E, Noguchi M, et al. International Association for the Study of Lung Cancer/American Thoracic Society/European Respiratory Society international multidisciplinary classification of lung adenocarcinoma. J Thorac Oncol 2011; 6: 244-285.

3 Weir BA, Woo MS, Getz G, et al. Characterizing the cancer genome in lung adenocarcinoma. Nature 2007; 450: 893-898.

4 Shields PG. Molecular epidemiology of smoking and lung cancer. Oncogene 2002; 21: 6870-6876.

5 Hung RJ, McKay JD, Gaborieau V, et al. A susceptibility locus for lung cancer maps to nicotinic acetylcholine receptor subunit genes on 15q25. Nature 2008; 452: 633-637.

DOI: 10.1183/09031936.00219811 